\title{
Estimation of Constant Speed Time for Railway Vehicles by Stochastic Gradient Descent Algorithm
}

\author{
Mehmet Taciddin Akçay ${ }^{1}$ \\ ${ }^{1}$ Istanbul Metropolitian Municipality, Directorate of Rail Systems, Istanbul, Turkey, \\ taciddin.akcay@ibb.gov.tr
}

Received 5 October 2020; Accepted 17 December 2020; Published online 30 December 2020

\begin{abstract}
While the investments in rail transportation systems continue without slowing down, various optimization issues come to the fore in order for the systems to work more efficiently. One of the most important of these issues is the optimization of the vehicle speed profile. Improvement in vehicle speed profile increases efficiency in operating traffic. Vehicle speed profile varies depending on the electrical-characteristic features of the vehicle, the distance between the stations and the line geometry. The vehicle's speed profile consists of several parts, such as acceleration, constant speed travel and braking zones. The constant speed in the constant velocity zone refers to the max operating speed, which is recommended for operation in the restricted area and remains within the limits. This part is critical in creating the speed profile of the vehicle. In this study, the estimation of the value of the constant speed time in the speed profile of the vehicles used in the city metro systems was made by using the Stochastic Gradient Descent method, which is one of the machine learning methods, and compared with various well-known methods. Coefficient of determination $\left(\mathrm{R}^{2}\right)$ values were calculated as 0.9955 and 0.9951 , respectively, with random sampling hold out and cross validation methods.
\end{abstract}

Keywords: Constant, railway, speed, stochastic, vehicle.

\section{Introduction}

Today, optimization issues for all subsystems are specifically addressed to use systems more effectively and efficiently. Rail transportation systems contain many systems. Since electrification systems form the main body in electric rail transportation systems, increasing the efficiency of this part is of great importance for the entire system. The energy consumption of the draw frame system in the electrification system corresponds to $50 \%$ of the total consumption. In the traction power system, the energy consumption of the rail system vehicle is the main element. The energy consumption of the vehicle varies depending on the traction power force that forms the vehicle's electrical power consumption curve. Figure 1 shows the variation of the traction power force of a rail vehicle based on the vehicle speed. The change of a 5 MVA rail system vehicle with a maximum speed of $140 \mathrm{~km} / \mathrm{h}$ is expressed.

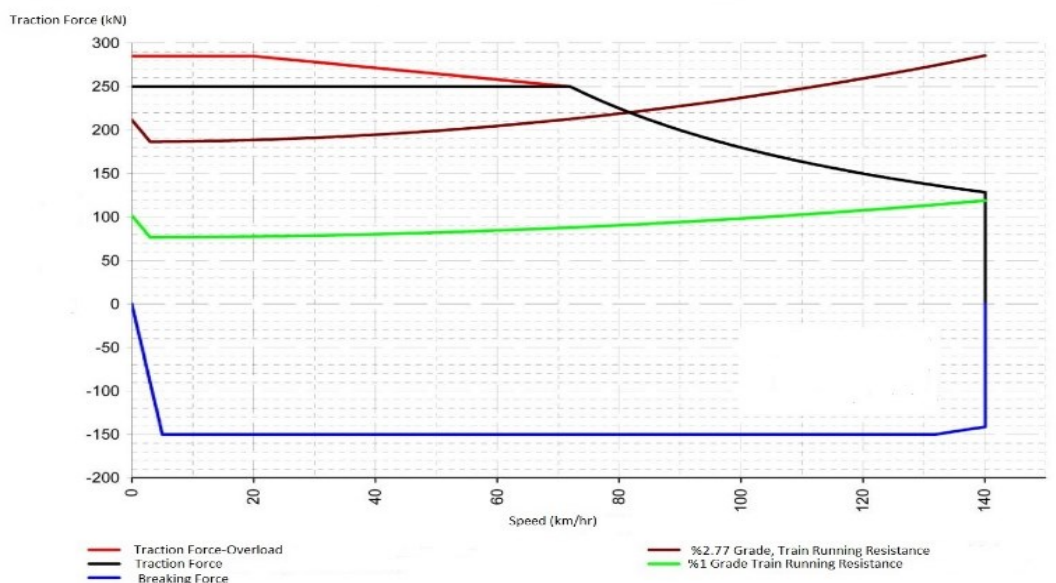

Figure 1 Traction power force- speed Graphic [Skoda] 
The red curve indicates the traction force it applies while accelerating the vehicle in the event of overload, while the black curve indicates the traction force in the acceleration conditions of the nominal condition. The blue curve shows the traction force of the vehicle in braking situation. With burgundy and green curves, the change of characteristic motion resistances of the vehicle corresponding to the slots of $2.7 \%$ and $1 \%$ is expressed. There are three different driving situations in vehicles such as acceleration, constant speed driving and braking. While defining the vehicle driving curve that will take place between the access points to the signaling system, the details of these driving modes are given. As seen in Figure 1, the point where the traction force is minimum is the constant speed point when the vehicle reaches the maximum speed in the driving modes before the vehicle is in braking state. Therefore, constant speed time in the vehicle's driving curve is critical when creating the driving mode. Figure 2 shows the region of the constant speed time in the vehicle's driving modes.

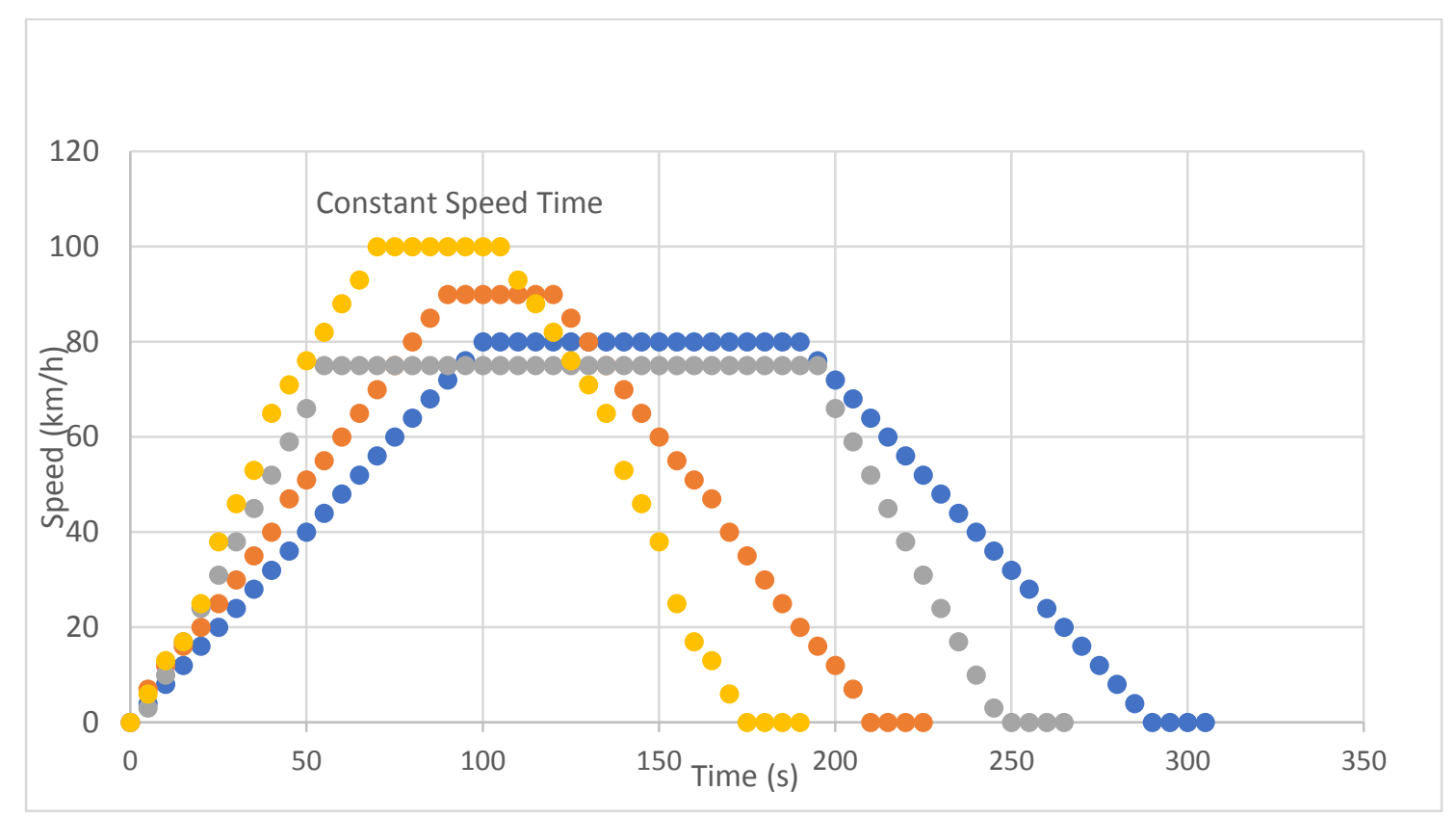

Figure 2 Graphic of vehicles driving modes

With this graphic, the speed profiles of the vehicles with different driving curves are given, and the maximum speed that each vehicle reaches, the constant speed traveled, and the acceleration applied while accelerating and decelerating vary. When searching the source, it is determined that there are many works on vehicle speed estimation. There are also sources where different machine learning algorithms are used and recommended. In the study [1], obtaining vehicle speeds with a simulator designed by using algorithms produced for rail system vehicle movements is explained. In [2], the subject of the measurement of the speed of the railroad vehicle and the regression models and the estimated time of arrival were investigated. In [3], the vehicle control model is proposed with the prediction of train movements and speed. With [4], the subject of calculating the movement resistance with the vehicle data collection mechanism has been examined. In [5], the help of dynamic time warping algorithms, the estimation of rail system vehicle speeds was investigated. In [6], with the proposed new method called ETL (EBER Track Lab), the subject of predicting the critical speed on the soft ground railway line was studied. Vehicle movements are simulated with the element increment method in [7]. In [8], the prediction of train motion resistance with the measurements in the on-board telemetric unit in the vehicles was investigated. With [9] the development of a prediction-controlled train business with simulation was studied. Control is provided via fixed and moving block. In [10], the subject of real-time arrival estimation for light rail systems was investigated. In the study [11], the accuracy of the traffic speed prediction was increased by using a new model named LC-RNN. In [12], speed estimation models of mixed traffic conditions in urban arteries were investigated. With [13] the equipment placed in commercial vehicles, cruise speed estimation was carried out by data collection method from mobile locations. In the article [14], vehicle speed estimation models are investigated within the road design depending on the energy demand. [15], the average speed estimation of the vehicle was investigated on 
a two-lane highway depending on weather and traffic characteristics. In [16], the subject of cruise speed estimation was examined using machine learning methods. Estimation methods of vehicle speed and driving modes have important advantages for the business. Real-time forecasts reduce navigational and operational uncertainties [17]. Determining the speed of the railway vehicle is also critical for railway operators and researchers working in the field of noise and vibration [18]. Vehicle driving modes affect the vehicle speed profile, and an optimization provided in this section reduces energy consumption and improves driving behavior. The constant speed time, which often takes place at maximum speed, is critical in achieving the best driving profile between the two access points. The efficiency to be achieved in the driving profile is of great importance for the benefit to be provided in the overall performance of the signaling system and the system. The fact that the maximum speed time can be predicted depending on the operation variables also increases the success of the operation traffic in the fleet management. In this study, which was accompanied by this information, estimation of the constant speed value between stations of the urban metro systems was made using the Stochastic Gradient Descent method, which is one of the machine learning methods.

\section{Material and Method}

\subsection{Simulation Model and Experimental Study}

With the test setup created in this study, 480 data sets with different operating conditions were obtained. Running resistance, Properties of Line Geometry, Traction force, Maximum Speed, Weight of the vehicle and Two Station Distance data are the entries of the system while the maximum constant speed time at the output is recorded. It is given by the equation (1) of the traction force applied by the vehicle while driving. This equation statement is the most basic formula used for the movement of the vehicle in the literature.

$$
\boldsymbol{F}_{\text {traction }}=\boldsymbol{F}_{\text {motion }}+\boldsymbol{F}_{\text {slope }}+\boldsymbol{F}_{\text {curve }}+\boldsymbol{m a}
$$

When $\mathrm{F}_{\text {traction }}$ indicates the traction power force, acceleration is expressed by a. $\mathrm{F}_{\text {motion }}, \mathrm{F}_{\text {slope }}, \mathrm{F}_{\text {curve }}$ are the forces acting on the vehicle during the travel, and $\mathrm{F}_{\text {motion }}$ refers to the force generated against the movement of the vehicle. $\mathrm{F}_{\text {slope }}$ and $\mathrm{F}_{\text {curve }}$, on the other hand, are related to line geometry and are forces that occur depending on slope and curve conditions. The weight of the vehicle is shown in $\mathrm{m}$. For the experimental setup, the system was operated by simulating the course of the vehicle for each operating situation. Figure 3 shows the screenshot of the experimental setup.

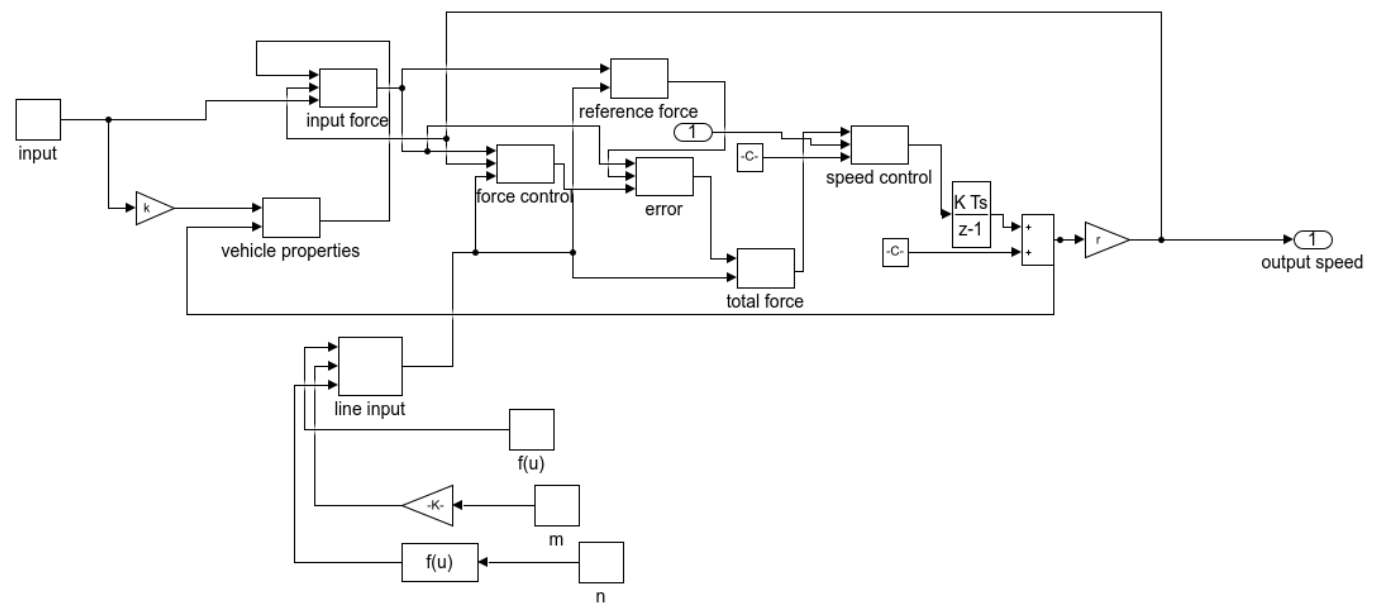

Figure 3 Experimental Study

Figure 4 shows the distributions of the data used. The success of the forecast results increases in proportion to the success of the data sets used here. Input 1 access distance, Input 2 Train resistance, Input 3 vertical resistance, input 4 lateral resistance, input 5 vehicle traction force, input 6 maximum speed, input 7 weight and output is constant speed time. Depending on the traction power line 
characteristics, the speed profile is achieved while accelerating the vehicle and the desired speed profile is captured. Speed is obtained as the output value in the rate of sensitivity entered. While the line features consist of line geometries such as slope and curve, the train resistance indicates a characteristic value related to the production of the vehicle. Although the vehicle weight is a value that varies according to the occupancy rate, mostly AW3 (6 passengers per square meter) value is used in the calculations. While the commercial speed value of the vehicle changes between $30-50 \mathrm{~km} / \mathrm{h}$ in the urban metro stations, the maximum speed value reached by the vehicle in operation can be much higher than this value.
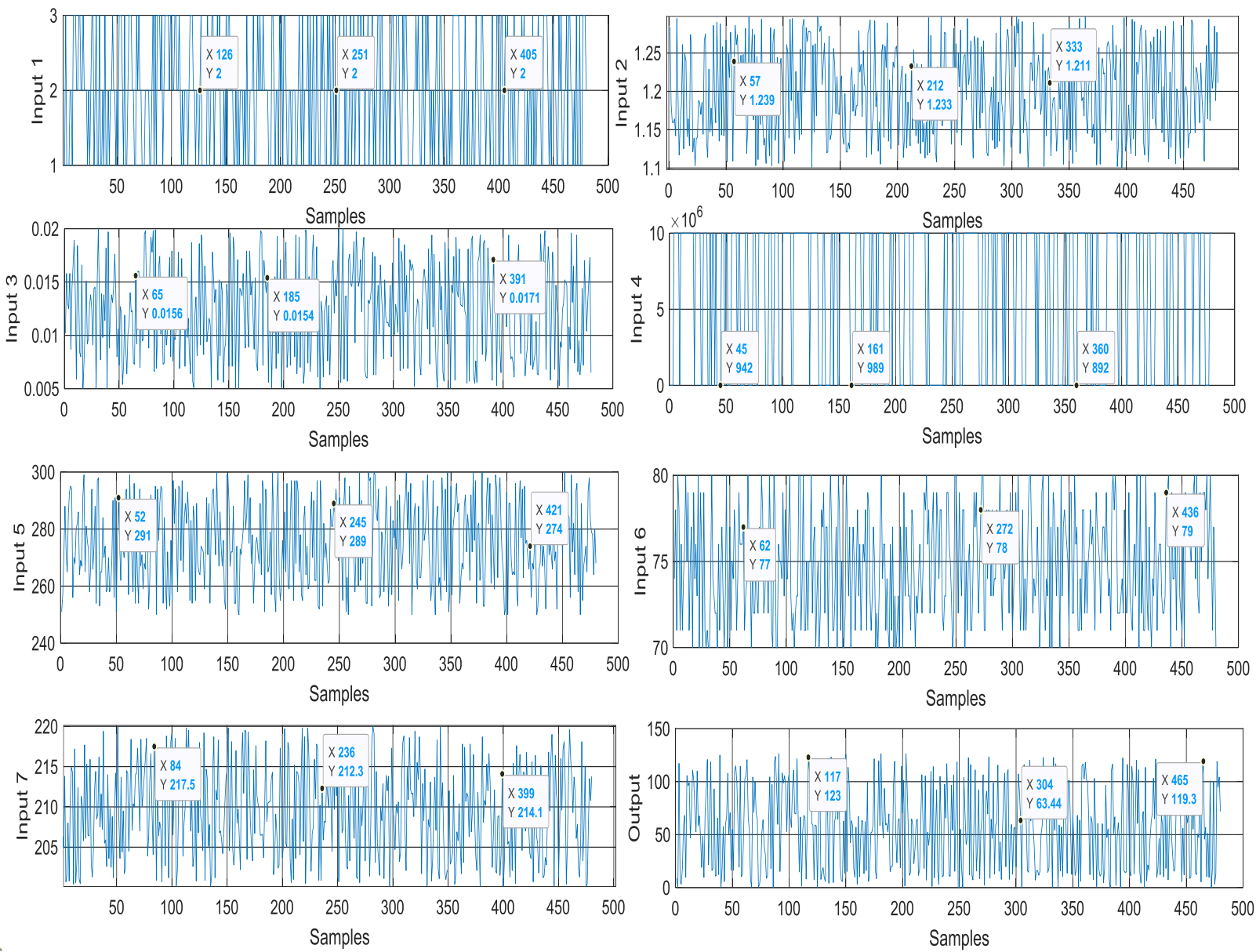

Figure 4 Distribution of input and output parameters

In order to obtain the essential sensitivity for the simulation, the sampling time was chosen at the appropriate value by considering the simulation speed. Machine learning methods are suitable for this analysis due to the variety of parameters depending on the operational conditions.

\subsection{Stochastic Gradient Descent}

Stochastic gradient descent (SGD) is a gradient descent optimization strategy used to minimize certain kinds of objective functions that arise in big data machine learning problems like building recommender systems [19]. SGD is the most popular family of optimisation algorithms used in machine learning on big data sets because of its ability to optimise efficiently with respect to the number of complete training set data epochs used [20]. SGD includes a parameter called the learning rate to define the length of the next step to take when moving forward in the direction of the gradient [20]. Choosing a accurate learning rate is a research field in itself and several contributions exist in this context [20]. Although some properties of SGD approaches might prevent their successful application to some optimization domains, they are well established in the machine learning community [21]. The application of SGD method is given by algorithm 1 [21]. Here are examples $X=\{\mathrm{x} 0, \ldots, \mathrm{xm}\}$ expresses iterations $T$, step interval $\epsilon$, and state variable $\mathrm{w}$. 


Algorithm 1 The Stochastic Gradient Descent Algorithm
\begin{tabular}{|c|c|}
\hline Step 1 & Given $\epsilon>0$ \\
\hline Step 2 & For $t=0, \ldots, T$ : \\
\hline Step 3 & Evaluate $\mathrm{j} \in\{1 \ldots \mathrm{m}\}$, randomly \\
\hline Step 4 & Update: $\omega_{T+1} \leftarrow \omega_{T}-\epsilon \partial_{\omega} x_{j}\left(\omega_{T}\right)$ \\
\hline Step 5 & Return output $\omega_{T}$ \\
\hline
\end{tabular}

The SGD algorithm operates in rounds; in each round, it traverses all edges in some order and updates labels at the end-points of each edge using a computation. The convergence factor in the SGD is investigated with the theories of convex minimization and of stochastic approximation. A very small learning rate cause with a slow convergence, however a large learning rate may affect convergence negatively and give rise to the loss function to fluctuate around the minimum value or may diverge [20]. Whether the small termination features of algorithms have a big practical effect convergence speed is an critical factor in large scale machine learning applications [21].

\subsection{Performance Calculations}

The success of the model proposed in the study was demonstrated by performance measurements. Mean Squared Error (MSE), Mean Absolute Error (MAE), Root Mean Square Error (RMSE) and Correlation Coefficient $\left(\mathrm{R}^{2}\right)$ measurements were used for performance calculations. The formulas of the relevant measurements are shown in Table 1.

Table 1 Performance Measures

\begin{tabular}{|c|c|c|c|}
\hline MSE & $\frac{1}{a} \sum_{i=1}^{a}\left(k_{\mathrm{i}}-m_{\mathrm{i}}\right)^{2}$ & RMSE & $\sqrt{\frac{1}{a} \sum_{i=1}^{a}\left(k_{\mathrm{i}}-m_{\mathrm{i}}\right)^{2}}$ \\
\hline MAE & $\frac{1}{a} \sum_{i=1}^{a}\left|k_{\mathrm{i}}-m_{\mathrm{i}}\right|$ & $\mathbf{R}^{2}$ & $1-\frac{\sum_{l=1}^{a}\left(k_{\mathrm{i}}-m_{\mathrm{i}}\right)^{2}}{\sum_{l=1}^{a}\left(k_{\mathrm{i}}-m_{a v g}\right)^{2}}$ \\
\hline
\end{tabular}

In formulas, $k_{i}$ and $m_{\mathrm{i}}$ are the estimated output values for the $\mathrm{i}^{\text {th }}$ desired result, respectively. The average of the desired output is shown in $m_{\text {avg }}$. The number of samples in the data series is indicated by a. These calculations were used as a reference when making comparisons about the results obtained.

\section{Results and Discussion}

In this research, the model designed with the proposed estimation method with 7 inputs and 1 output was applied to the data obtained as a result of the rail system test studies using the Orange machine learning program. Decision Trees, Adaboost, Neural Network (NN), SVM, kNN, Random Forest (RF) and Stochastic Gradient Descent methods, which are widely used in the literature, are studied. In this study, stochastic gradient descent method is especially recommended due to the success obtained. Figure 5 shows the designed architecture of the system.

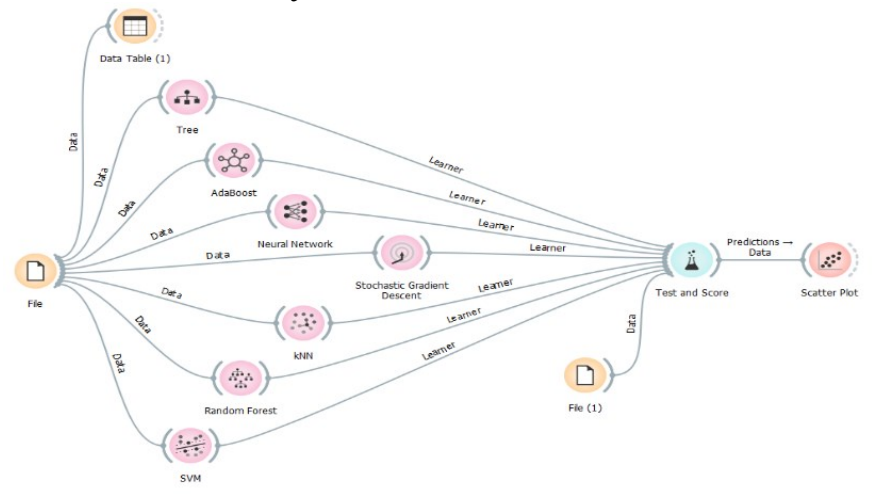

Figure 5 Architecture of the system 
The results are given by examining the designed model with cross validation and random sampling hold out methods separately. While the data was divided into 10 groups with the cross validation method, $70 \%$ of the data was used in education in the random sampling hold out method. The remaining $30 \%$ was used for testing. In the cross validation method, the training / test ratio was repeated for each cycle with a 9: 1 ratio.

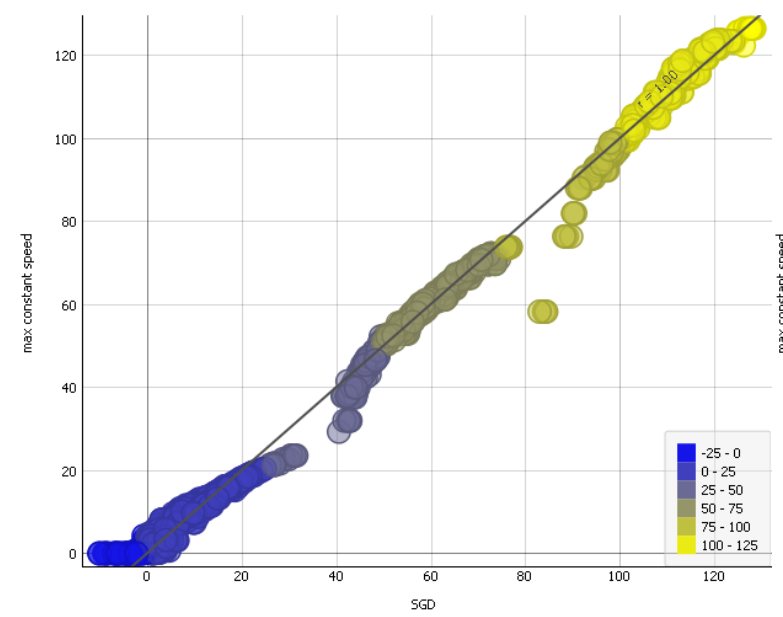

(a) Cross validation

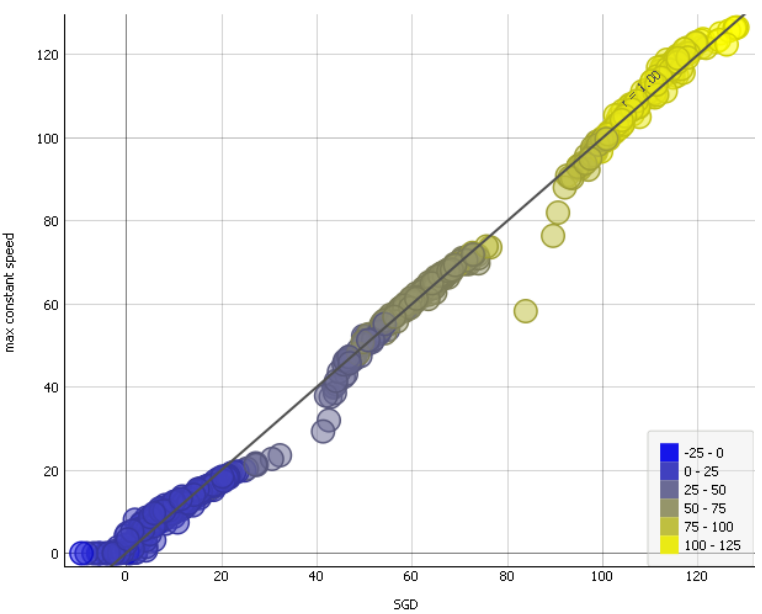

(b) Random sampling

Figure 6 SGD Regression Graphs

The results obtained for different methods are given in Table 2. The SGD method proposed in this study and the most successful results are obtained, and the performance values of AdaBoost, Random Forest, Neural Network, kNN, Decision Trees and SVM methods, which are frequently used in the literature, can be seen in these tables, respectively.

Table 2 Comparison of the Estimated Performance of the Methods Used

\begin{tabular}{|l|r|r|r|r|}
\hline \multicolumn{5}{|c|}{ Cross Validation } \\
\hline Method & \multicolumn{1}{|c|}{ MSE } & RSE & \multicolumn{1}{l|}{ MAE } & R $^{\mathbf{2}}$ \\
\hline $\begin{array}{l}\text { Stochastic } \\
\text { Gradient Descent }\end{array}$ & 6.8174458902 & 2.6110239160 & 1.7105567557 & 0.9955774039 \\
\hline AdaBoost & 9.9926887499 & 3.1611214386 & 2.1115000000 & 0.9935175685 \\
\hline Random Forest & 9.6631255899 & 3.1085568339 & 2.1420637041 & 0.9937313618 \\
\hline Neural Network & 20.488570730 & 4.5264302414 & 3.3677543843 & 0.9867087067 \\
\hline kNN & 161.69644876 & 12.715991851 & 9.6273916666 & 0.8951046932 \\
\hline Tree & 15.085633926 & 3.8840229049 & 2.7802256944 & 0.9902136861 \\
\hline SVM & 184.92898709 & 13.598859771 & 11.543075796 & 0.8800333404 \\
\hline \multicolumn{5}{|c|}{ Random Sampling } \\
\hline Stochastic & 7.6042002437 & 2.7575714394 & 1.7628956167 & 0.9951452581 \\
Gradient Descent & 11.173792499 & 3.3427223187 & 2.2163472222 & 0.9928663270 \\
\hline AdaBoost & 11.871545564 & 3.4455109293 & 2.3395522473 & 0.9924208612 \\
\hline Random Forest & 40.502361962 & 6.3641466013 & 4.9793130202 & 0.9741421183 \\
\hline Neural Network & 204.00158543 & 14.282912358 & 11.174386111 & 0.8697594758 \\
\hline kNN & 18.001537177 & 4.2428218413 & 3.0273217592 & 0.9885072969 \\
\hline Tree & 230.87452593 & 15.194555799 & 12.781131179 & 0.8526030118 \\
\hline SVM & & & & \\
\hline
\end{tabular}

When Cross Validation and Random sampling methods are examined separately, it is seen that the best success is achieved with the proposed method Stochastic Gradient Descent method. The success order in cross validation method is Random Forest, AdaBoost, Decision Trees, Neural Network, kNN, and SVM. When calculations are made with random sampling, the best methods are AdaBoost, Random Forest, Decision Trees, Neural Network, kNN, and SVM, respectively. Figure 7 and Figure 8 show error graphs of the estimates obtained by SGD cross Validation and SGD Random Sampling methods. 


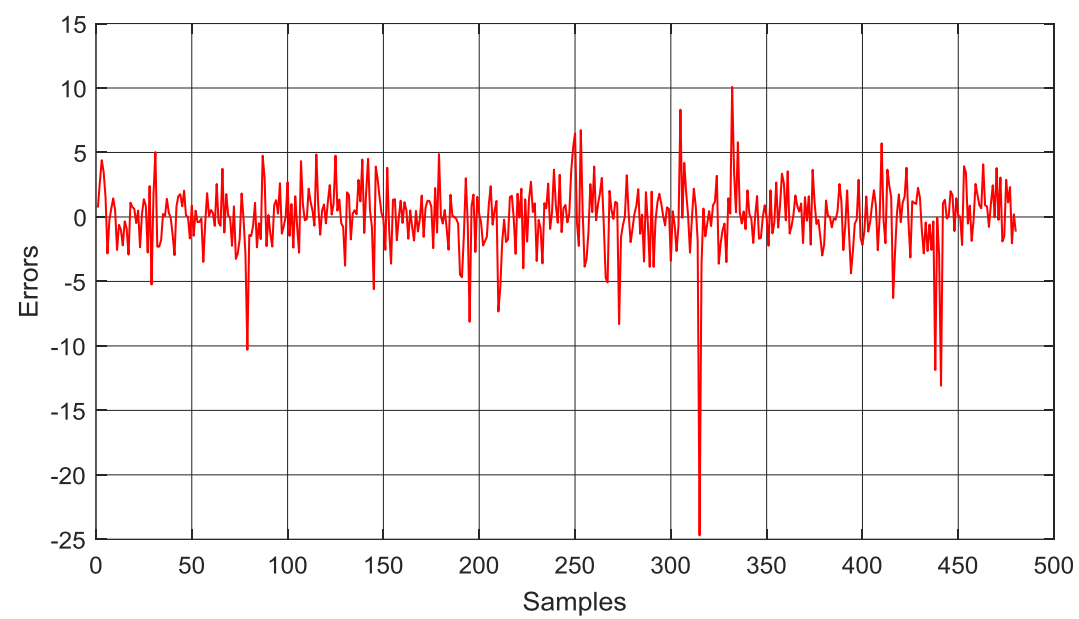

Figure 7 Errors for Cross Validation

In the Random Sampling method, 144 data corresponding to $30 \%$ of the total data were used for the test.

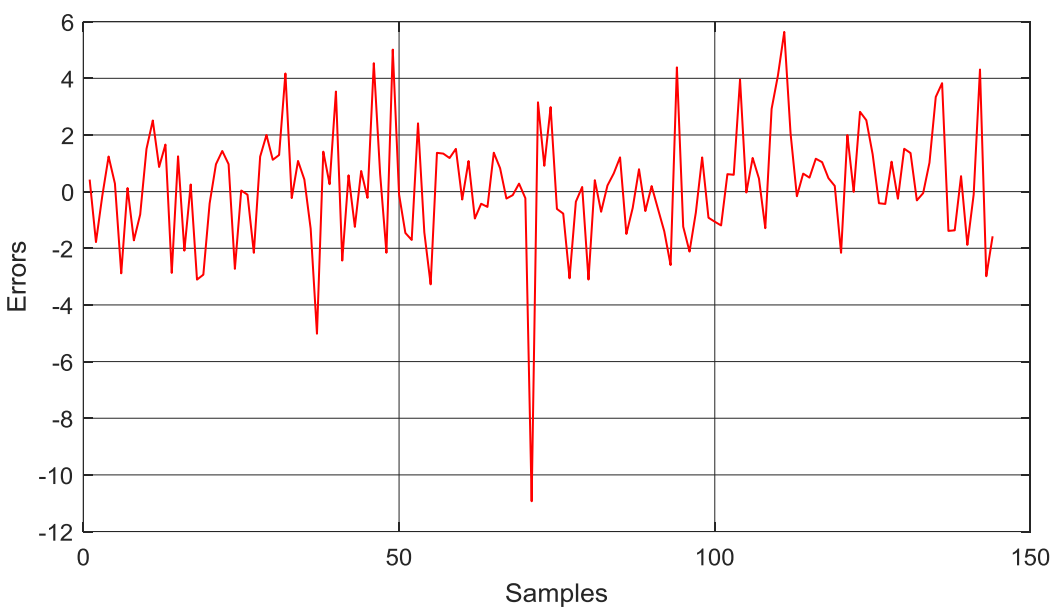

Figure 8 Errors for Random Sampling

The amount of error in the cross validation method deviated more than the Random sampling method. In Figure 9, the regression graphs, which compare the estimates produced using the SGD Cross Validation method and other methods, were created for each method, respectively. While the horizontal axis consists of SGD results, the vertical axis is formed from the results of other methods.

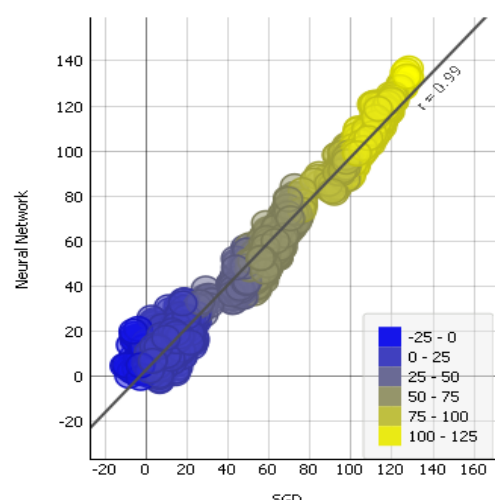

(a) SGD-NN

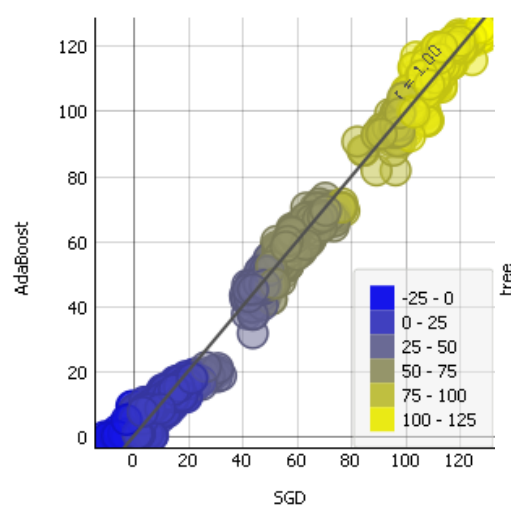

(b) SGD-AdaBoost

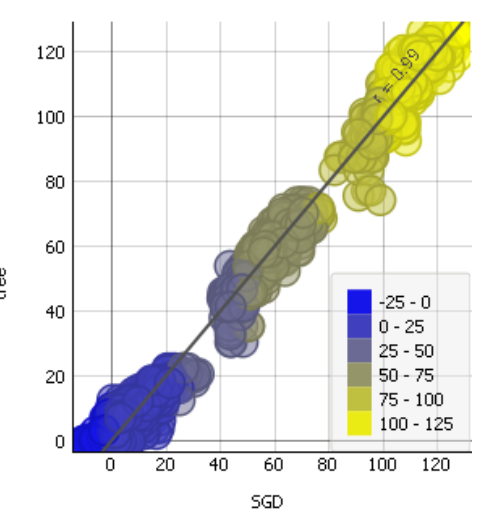

(c) SGD-tree

Figure 9 SGD/Cross Validation Regression Graphs with Other Methods 


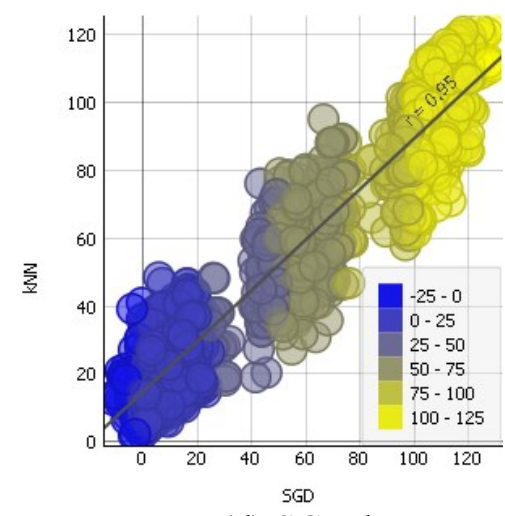

(d) SGD-kNN

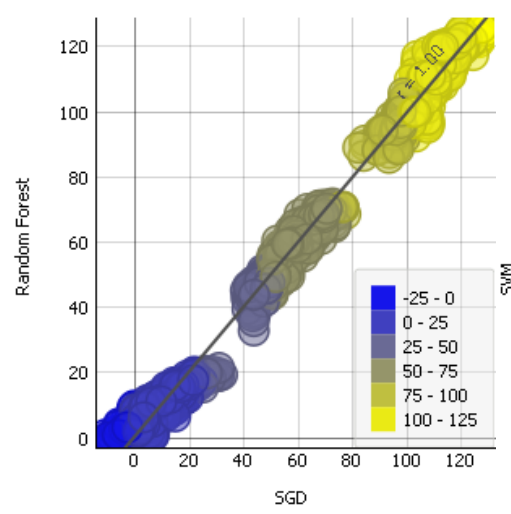

(e) SGD-Random Forest

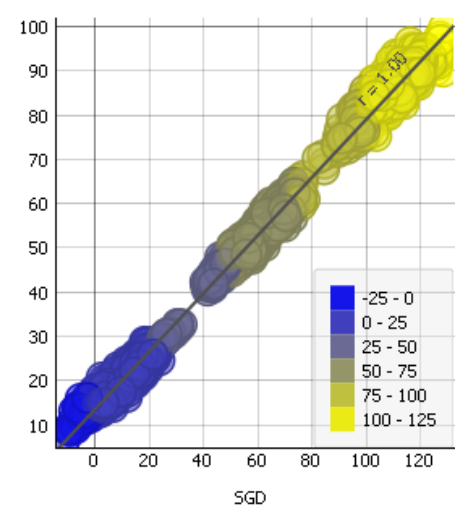

(f) SGD-SVM

Figure 9 SGD/Cross Validation Regression Graphs with Other Methods (Continued)

As seen in the graphs given in Figure 9, the regression value of AdaBoost, Random Forest and SVM methods is 1 , while this value is 0.99 in Neural Network and Decision Trees. In the kNN method, the regression value is 0.95. In Figure 10, this situation was applied for SGD Random Sampling method and similar graphics were produced.

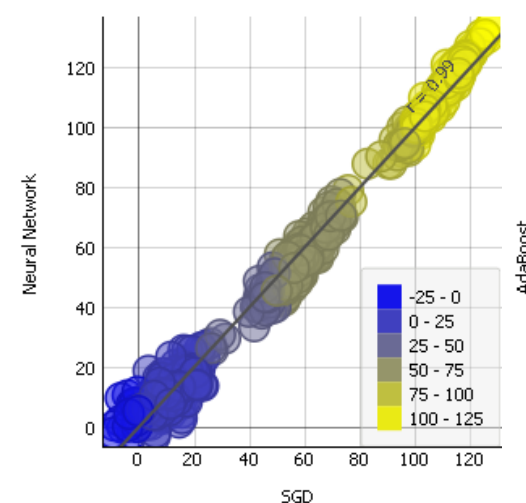

(b) SGD-NN

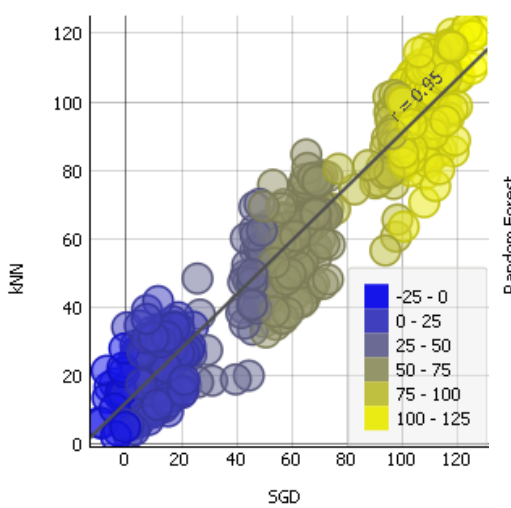

(d) SGD-kNN

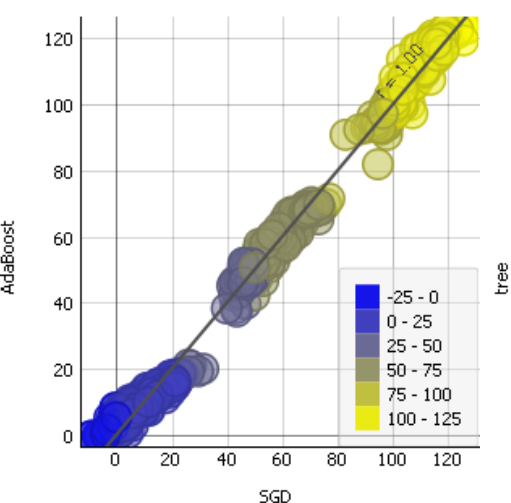

(b) SGD-AdaBoost

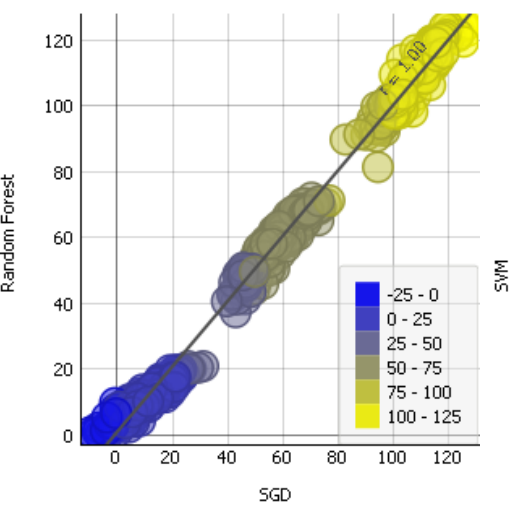

(e) SGD-Random Forest

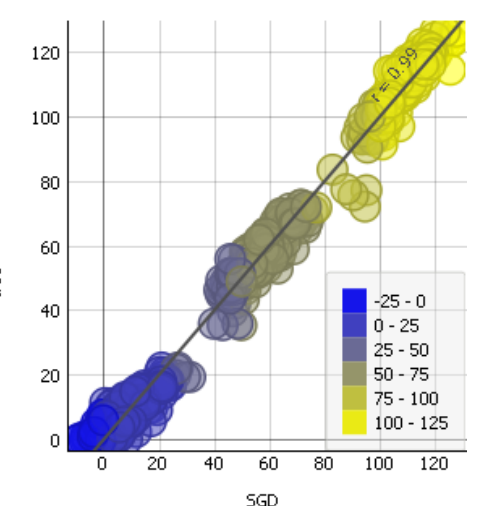

(c) SGD-tree

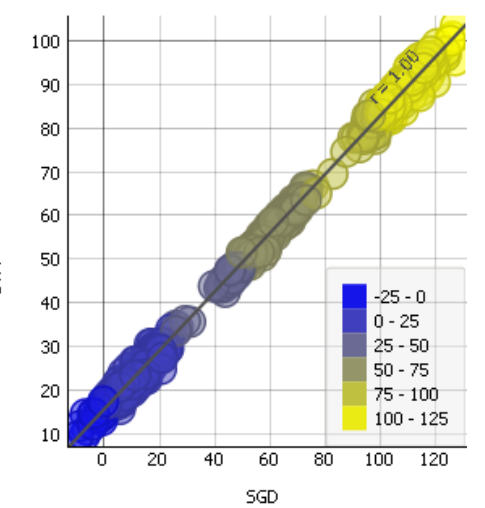

(f) SGD-SVM

Figure 10 SGD/Random Sampling Regression Graphs with Other Methods

Looking at the graphs in Figure 10, the success rate of 0.99 and above is achieved in all methods except $\mathrm{kNN}$, while the regression value of $\mathrm{kNN}$ results is 0.95 . In the graph indicated by (d) in Figure 10, this is understood by the deviation of the data by more than the 45 degree curve. When the values given in Table 2 are examined, MSE value is 6.82, RMSE value is 2.61, MAE value is 1.71 and R2 value is 0.9955 with SGD Cross Validation method. In Random Sampling method, MSE value is 7.6, RMSE value is $2.76, \mathrm{MAE}$ value is $1.76, \mathrm{R} 2$ value is 0.9951 . With the algorithm produced in [1], the arrival time of the trains was estimated with an average difference of $0.16 \%$. In [2], 90\% success was achieved 
in the results obtained by the kinematic method used for estimation. Success was achieved with a delay of 12.3 seconds with the prediction-based control method proposed in the study with [3]. In [4], there was a $0.1 \%$ difference between the values measured by the train motion resistance obtained using the data provided by the test method. In study [6], it was explained that the critical speed was estimated with a difference of $15 \mathrm{~km} / \mathrm{h}(250-235)$. In the study with [7], simulation of the train movement was provided by optimizing the vehicle driving curve. In the study [8], the train movement resistance was estimated between $1.1 \%$ and $12.1 \%$, depending on the vehicle speed. With [9], a simulation of 2 to 5 seconds has been achieved for the frequency of vehicle voyages in the prediction controlled train operation. In study [10], it was stated that the difference between the results obtained by the AVLS (Automatic Vehicle Location System) and the VBA (Visual Basic for Applications) methods did not exceed 25 seconds.

\section{Conclusion}

Constant speed time is critical for the efficiency of the rail system signaling system and effective management in operating traffic. Due to the many dynamic elements it contains within the rail system operation, the system reacts rapidly to changing conditions and activation of the system as desired makes a great contribution to the system. With this study, the constant speed time between the access points in rail systems has been estimated by machine learning methods. At the end of this study, the constant speed time, which is critical in ensuring the frequency of voyage in the rail system signaling system, was estimated with high accuracy by applying the proposed method. The machine learning methods studied produced predictions with Cross Validation and Random Sampling techniques. Stochastic Gradient Descent, Random Forest, AdaBoost, Decision Trees, Neural Network, kNN, and SVM methods were applied to the constant speed estimates made using experimentally generated data sets. While the obtained results are given comparatively, the most successful results were obtained with the proposed method Stochastic Gradient Descent method. While the results of MSE, RMSE, MAE and R ${ }^{2}$ values calculated for performance evaluation are given in the table, the amounts of errors obtained are presented. With the proposed method, the performance of the vehicle traffic is increased by providing high efficiency in the rail system operation and the signalization system, where dynamic operating conditions are available. In this way, by providing flexibility in the system operating conditions, the most appropriate answer can be produced quickly and the situations brought by the results can be activated. This study is of great importance for increasing and promoting machine learning applications in rail system signaling system elements with high data sets.

\section{Acknowledgments}

We would like to thank Istanbul Metropolitan Municipality, Rail System Department, for its support during the realization of this study.

\section{References}

[1] J. Jong and S. Chang, "Algorithms for generating train speed profiles," Journal of the Eastern Asia Society for Transportation Studies, no.6, pp. 356-371, 2005.

[2] Y. Chen and L.R. Rilett, "A train speed measurement and arrival time prediction system for highway-rail grade crossings," Transportation Research Record Journal of the Transportation Research Board, vol. 2608, no.1, pp. 96-104, 2017.

[3] S. Hiraguri, "Evaluation of Train Control Method Using Prediction Control," Quarterly Report of Rtri, vol. 49, no. 3, pp. 163-167, 2008. 
[4] T. Ogawa, S., Manabe, G. Yoshikawa, Y. Imamura and M. Kageyama, "Method of Calculating Running Resistance by the Use of the Train Data Collection Device," Quarterly Report of RTRI, no.1 58, pp. 21-27, 2017.

[5] S. Hensel and M. Marinov, "Time Signal Based Warping Algorithms for Low Speed Velocity Estimation of Rail Vehicles," Annual Journal of Electronics, no. 8, pp. 177 - 180, 2014.

[6] K. N. Cosgriff, E. G. Berggren, A. M. Kaynia, N. N. Dam and N. Mortensen, "A new method for estimation of critical speed for railway tracks on soft ground," International Journal of Rail Transportation, vol. 6, no. 4, pp. 203-217, 2018.

[7] G., Xu, F., Li, J., Long, D., Han, "Train movement simulation by element increment method", Journal of advanced transportation, no. 50, pp. 2060-2076, 2017.

[8] S., Aradi, T., Becsi, P., Gaspar, "Estimation of running resistance of electric trains based on onboard telematics system", International Journal of Heavy Vehicle Systems, no. 22, pp. 277-291, 2015.

[9] T., Kunimatsu, T., Terasawa, Y., Takeuchi, "Evaluation of Train Operation with Prediction Control by Simulation", in International Conference on Railway Operations Modelling and Analysis, 2019, pp.589-606.

[10] E. Naye, "Real-time arrival prediction models for light rail train systems," Royal Instistute of Technology, Department of Engineering, Master's Thesis, 2014.

[11] Z. Lv, J. Xu, K. Zheng, H. Yin, P. Zhao and X. Zhou, "LC-RNN: A Deep Learning Model for Traffic Speed Prediction," in Proceedings of the Twenty-Seventh International Joint Conference on Artificial Intelligence (IJCAI-18), pp. 3470-3476, 2018.

[12] A. Dhamaniya and S. Chandra, "Speed Prediction Models for Urban Arterials under Mixed Traffic Conditions," Procedia - Social and Behavioral Sciences, no. 104, pp. 342 - 351, 2013.

[13] M. Gmira, M. Gendreau, A. Lodi and J., Potvin, "Travel Speed Prediction Based on Learning Methods For Home Delivery," Interuniversity Research Center On Business Networks, logistics and transport, pp. 1-34, 2018.

[14] M. Bysveen, "Vehicle speed prediction models for consideration of energy demand within road design," Norwegian University of Science and Technology, Civil and Environmental Engineering, Master's Thesis, 2017.

[15] B. Mirbaha, M. Saffarzadeh, S. A. Beheshty, M. Aniran, M. Yazdani and B. Shirini, "Predicting Average Vehicle Speed in Two Lane Highways Considering Weather Condition and Traffic Characteristics," IOP Conference Series: Materials Science and Engineering, pp. $1-7,2017$.

[16] M. Gmira, M. Gendreau, A. Lodi and J. Potvin, "Travel speed prediction using machine learning techniques," ITS World Congress, pp. 1-10, 2017.

[17] O. Cats, "Real-Time Predictions for Light Rail Train Systems," 17th International IEEE 
Conference on Intelligent Transportation Systems (ITSC), pp. 1-10, 2014.

[18] G. Kouroussis, D. P. Connolly, M. Forde and O. Verlinden, "Train speed calculation using ground vibrations," Proceedings of the Institution of Mechanical Engineers, Part F: Journal of Rail and Rapid Transit, vol. 229, no. 5, pp. 466-483, 2015.

[19] R. Kaleem, S. Pai and K. Pingali, "Stochastic Gradient Descent on GPUs," ACM International Conference Proceeding Series, pp. 81-89, 2015.

[20] I. Chakroun, T. Haber and T. Ashby, "SW-SGD: The Sliding Window Stochastic Gradient Descent Algorithm," Procedia Computer Science, no. 108, pp. 2318-2322, 2017.

[21] J. Keuper (Fehr) and F. J. Pfreundt, "Asynchronous parallel stochastic gradient descent: a numeric core for scalable distributed machine learning algorithms," MLHPC '15: Proceedings of the Workshop on Machine Learning in High-Performance Computing Environments, pp. 1$11,2015$. 